\title{
EFFECT OF GRAIN SIZE ON SLOW FATIGUE CRACK PROPAGATION AND PLASTIC DEFORMATION NEAR CRACK TIP
}

\author{
R. DENG, G. YU and H. LI \\ Institute of Mechanics, Academic Sinica, Beijing, China
}

\begin{abstract}
Fatigue crack growth and its threshold are investigated at a stress ratio of 0.5 for the three-point bend specimen made of Austenitic stainless steel. The effect of grain size on the crack tip plastic deformation is investigated. The results show that the threshold value $\Delta k_{\text {th }}$ increases linearly with the square root of grain size $d$ and the growth rate is slower for materials with larger grain size. The plastic zone size and ratio $\left(k_{\max } / \sigma_{\mathrm{ys}}\right)^{2}$ for different grain sizes are different at the threshold. The maximum stress intensity factor is $k_{\max }$ and $\sigma_{\mathrm{ys}}$ is the yield strength. At the same time, the characteristics of the plastic deformation development is discontinuous and anti-symmetric as the growth rate is increased from $2 \cdot 10^{-8}$ to $10^{-7}$ $\mathrm{mm} /$ cycle.

A dimensionless relation of the form $n \Delta k_{\mathrm{th}} / 2 \sigma_{\mathrm{ys}} \sqrt{d}$ for collating fatigue crack starting growth data is proposed in which $\Delta k_{\text {th }}$ represents the stress intensity factor range at the threshold. Based on experimental results, this relation attains the value of 0.6 for a fatigue crack to start growth in the Austenitic stainless steel investigated in this work. Metallurgical examinations were also carried out to show a transgranular shear mode of cyclic cleavage and plastic shear.
\end{abstract}

\section{Introduction}

Material behavior is known to depend on its internal structure. A change in grain size, $d$ will affect the mechanical properties [1] and rate of fatigue crack growth. Investigated in [2] is the effect of ferritic grain size of low carbon steel on the threshold value, $\Delta k_{\mathrm{th}}$ with $\Delta k$ denoting the range of stress intensity factor. The result showed that the threshold increases with grain size. Contrary results, however, were obtained in [3] for the aluminium-copper alloy. Moreover, the value of $\Delta k_{\mathrm{th}}$ for the $2618 \mathrm{~A}$ aluminium alloy showed no dependence on $d$ [4].

The Austenitic stainless steel is used here to investigate the effect of face-centered cubic grain size on $\Delta k_{\text {th }}$ by varying the mechanical properties. Experimental observations of plastic deformation [5] and fractured surface by fatigue are also carried out.

\section{Experiment procedure}

Austenitic stainless steel with chemical composition $0.04 \% \mathrm{C}, 15.5 \% \mathrm{Cr}$ and $14.5 \% \mathrm{Ni}$ was treated by solution heat treatment at three different temperatures to obtain three different grain sizes: $0.181,0.143$ and $0.044 \mathrm{~mm}$. They are referred to as material A, B and C in Table 1 with their respective mechanical properties. In Table 1, $\sigma_{u}$ is the ultimate strength, $\sigma_{y s}$ the yield strength, $\delta \%$ the percentage elongation, $\psi \%$ the percentage reduction in area, $\mathrm{n}$ the hardening exponent.

All tests were carried out by using the threepoint bend specimens [5] at a frequency of $115 \mathrm{~Hz}$ and a stress ratio value of 0.5 . The plastic deformation at crack tip was directly observed by means of Phase Contract Microscope (PCM). In the test, the stress intensity factor range, $\Delta k$ defined as $\Delta k_{\text {th }}$, corresponds to load cycle, $N$ equals $10^{7}$ such that the increment in crack did not exceed $0.2 \mathrm{~mm}$.

Table 1

Mechanical properties of steel tested

\begin{tabular}{lllllll}
\hline Material & $\begin{array}{l}\sigma_{\mathrm{u}} \\
\left(\mathrm{MN} / \mathrm{m}^{2}\right)\end{array}$ & $\begin{array}{l}\sigma_{\mathrm{ys}} \\
\left(\mathrm{MN} / \mathrm{m}^{2}\right)\end{array}$ & $\begin{array}{l}E \\
\left(\mathrm{MN} / \mathrm{m}^{2}\right)\end{array}$ & $\delta \%$ & $\psi \%$ & $n$ \\
\hline A & 460.9 & 137.8 & 177506.7 & 67.6 & 80.4 & 0.45 \\
B & 464.8 & 139.7 & 190255.8 & 67.2 & 77.7 & 0.42 \\
C & 501.1 & 168.2 & 234387.3 & 73.0 & 82.6 & 0.39 \\
\hline
\end{tabular}




\section{Experimental results and analysis}

\subsection{Fatigue crack propagation}

Test results show that $\Delta k_{\text {th }}$ increases linearly with $\sqrt{d}$. The relation of $\Delta k_{\mathrm{th}}$ and $\sqrt{d}$ can be expressed as

$\Delta k_{\mathrm{th}}=4.8+0.275 \cdot 10^{2} \sqrt{d}$

Slow crack growth rate data are displayed in Figure 1 , showing the influence of grain size. The rate is slower for larger grain size. This effect becomes less pronounced as $\Delta k$ is increased.

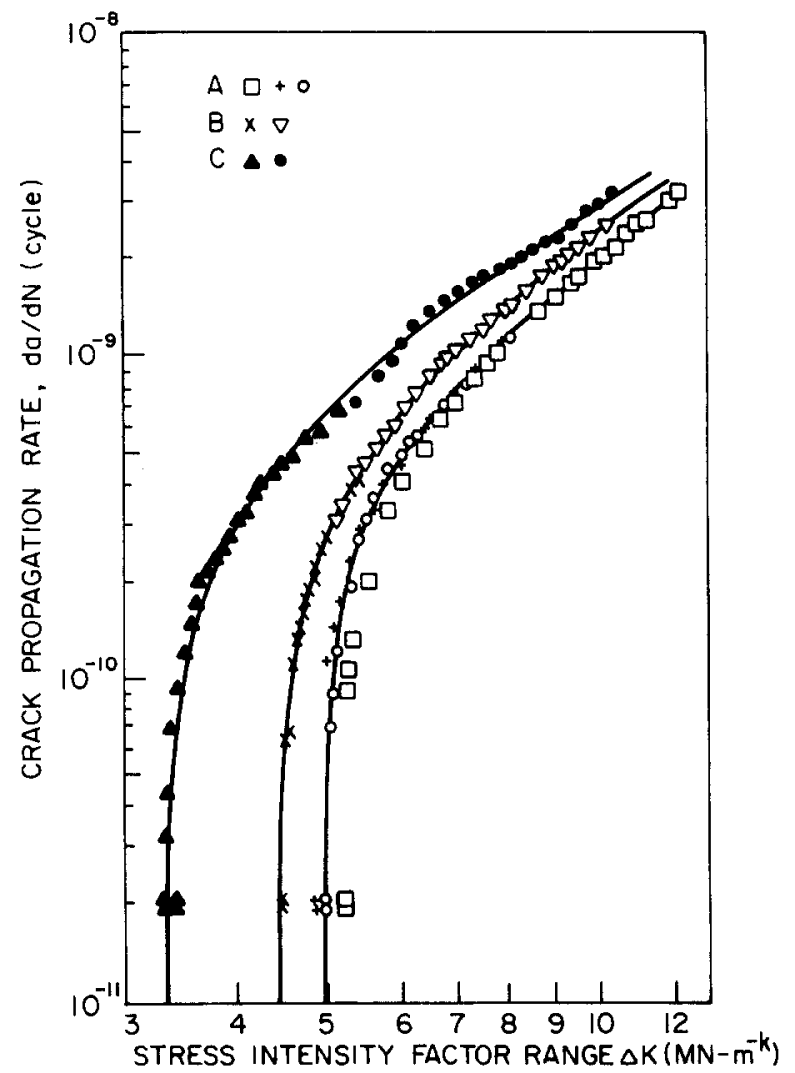

Fig. 1. Effect of grain size on crack growth rate.
A dimensionless relation of fatigue crack initiation is proposed:

$n \frac{\Delta k_{\mathrm{th}}}{\sigma_{\mathrm{ys}} \sqrt{d}}=$ const.

Equation (2) is approximately equal to 0.6 for all three grain sizes. Refer to the results in Table 2.

\subsection{Plastic zone observation}

The plastic deformation zone is observed by PCM and its size is measured at threshold for the three different grain sizes. The results are given in Table 3. In fatigue, the plastic zone of fatigue crack tip consists of the monotonic plastic zone and reversed plastic zone. Let $A_{x}$ and $A_{y}$ be the proportionality factors of the monotonic plastic zone size (MPZS) in the $x$ and $y$ directions and $\left(k_{\max } / \sigma_{\mathrm{ys}}\right)^{2}$. The corresponding MPZS are $R_{x}$ and $R_{y}$, and they are in units of $\mathrm{mm}$. At threshold, the subscript "th" will be added. Note that $R_{x}$ and $R_{\mathrm{y}}$ are larger for larger grains while the ratio $\left(R_{y}\right)_{\mathrm{th}} /\left(R_{x}\right)_{\mathrm{th}}$ remained relatively constant.

The characteristics of plastic deformation in front of the crack tip at threshold are:

(1) The single slip extend all over the plastic zone and the slip bands do not penetrate the grain. Refer to Fig. 2(a).

(2) The plastic deformation development is discontinuous and anti-symmetric at the threshold.

Table 3

Plastic zone size of crack tip along the lines of $x$ and $y$ at threshold

\begin{tabular}{llllll}
\hline Material & $\begin{array}{l}\left(R_{y}\right)_{\mathrm{th}} \\
(\mathrm{mm})\end{array}$ & $\begin{array}{l}\left(R_{x}\right)_{\mathrm{th}} \\
(\mathrm{mm})\end{array}$ & $\frac{\left(R_{y}\right)_{\mathrm{th}}}{\left(R_{x}\right)_{\mathrm{hh}}}$ & $\left(A_{\mathrm{y}}\right)_{\mathrm{th}}$ & $\left(A_{x}\right)_{\mathrm{th}}$ \\
\hline $\mathrm{A}$ & 0.487 & 0.180 & 2.71 & 0.046 & 0.034 \\
$\mathrm{~B}$ & 0.307 & 0.120 & 2.56 & 0.034 & 0.027 \\
$\mathrm{C}$ & 0.070 & 0.028 & 2.50 & 0.021 & 0.018 \\
\hline
\end{tabular}

Table 2

Dimensionless criterion of crack starting propagation

\begin{tabular}{|c|c|c|c|c|c|c|c|}
\hline Material & $\begin{array}{l}\sigma_{\mathrm{ys}} \\
\left(\mathrm{MN} / \mathrm{m}^{2}\right)\end{array}$ & $\begin{array}{l}\text { Average } \\
\Delta k_{\text {th }} \\
\left(\mathrm{MN} / \mathrm{m}^{3 / 2}\right)\end{array}$ & $\begin{array}{l}\sqrt{d} \\
(\sqrt{\mathrm{m}})\end{array}$ & $n$ & $n \frac{\Delta k_{\mathrm{th}}}{2 \sigma_{\mathrm{ys}} \sqrt{d}}$ & $\begin{array}{l}\text { Constant in } \\
\text { eq. (2) }\end{array}$ & $\begin{array}{l}\text { Error } \\
\%\end{array}$ \\
\hline $\bar{A}$ & 137.8 & 5.01 & 0.0135 & 0.45 & 0.6060 & & 1.05 \\
\hline $\mathbf{B}$ & 139.7 & 4.70 & 0.0120 & 0.42 & 0.5888 & 0.5997 & -1.81 \\
\hline $\mathrm{C}$ & 168.2 & 3.44 & 0.0066 & 0.39 & 0.6043 & & 0.76 \\
\hline
\end{tabular}



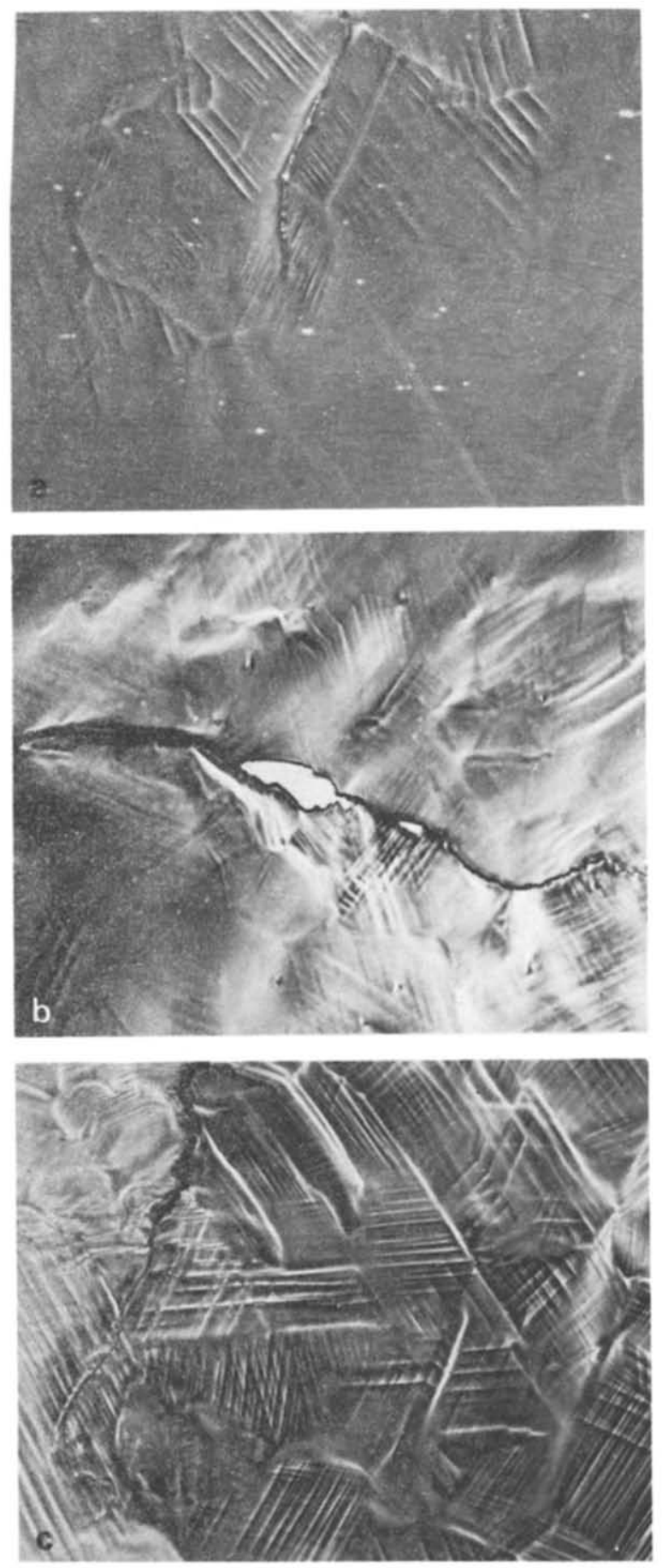

Fig. 2. Plastic deformation zone; (a) at $\Delta k_{\text {th }}$; (b) $\mathrm{d} a / \mathrm{d} N<$ $10^{-7} \mathrm{~mm} /$ cycle under PCM; (c) $\Delta k=14.1 \mathrm{MN} / \mathrm{m}^{3 / 2}$.

With crack growth rates of 1.0 to $5.0 \cdot 10^{-6}$ $\mathrm{mm} /$ cyc., $\left(A_{y}\right)_{\mathrm{th}}$ for the three different grain sizes are $0.082,0.080$ and 0.074 . Grain size effect is not observed. For $\mathrm{d} a / \mathrm{d} N<10^{-7} \mathrm{~mm} /$ cyc., the plastic deformation on both sides of crack surface is also discontinuous and anti-symmetric. The mechanism of plastic deformation is mainly a single slip, Fig. 2(b). Nonhomogeneity prevails at the microscopic level being of the order of grain size. The slip bands are observed in a limited number of regions, but the fine slip bands can only be seen by PCM.

As $\Delta k$ is increased, plastic deformation becomes more uniform and continuous. The slip bands become more dense as the mechanism of plastic deformation switches from that of single slip to a multiple-slip system (MSS), Fig. 2(c). The process is also sensitive to twin grain boundaries. Whenever a slip encounters a twin boundary, the direction of slip changes. Parallel slip bands are rare but they can appear near the elastic-plastic interface. The crack tip plastic zone spreads out to both sides of the crack at an angle. While zone size increases with $\Delta k$, their position tends to lean forward. This is observed for all three grain sizes. Fatigue fracture is mostly transgranular and the direction of growth is disturbed when encountering a twin boundary.

\subsection{Fracture surface}

Figure 3(a) shows that the fracture surface is predominantly transgranular regardless of grain size. The small facet is of the order of grain size and its orientation is affected by grain and twin boundaries, Fig. 3(b). Fatigue crack growth when examined at the microscopic level reveals fine striation that are approximately normal to the direction of macro-crack growth. Discontinuity and anti-symmetry of plastic deformation near the crack tip are due to the influence of material microstructure. Shear along the direction of slip tends to enhance plastic deformation. This depends on the local intensity of the crack tip stress field. Substructure of the material can also be an obstacle to slip in addition to twin or grain boundaries themselves. The observed result is different from that introduced in [6]. In general, multiple slip tends to dominate when the local stress intensity is elevated where dense cross slip bands can be more readily formed. Reverse loading can also enhance multiple slip. Parameters $A_{y}$ and $A_{x}$ not only depend on material but also on the intensity of the stress field of crack tip and is 

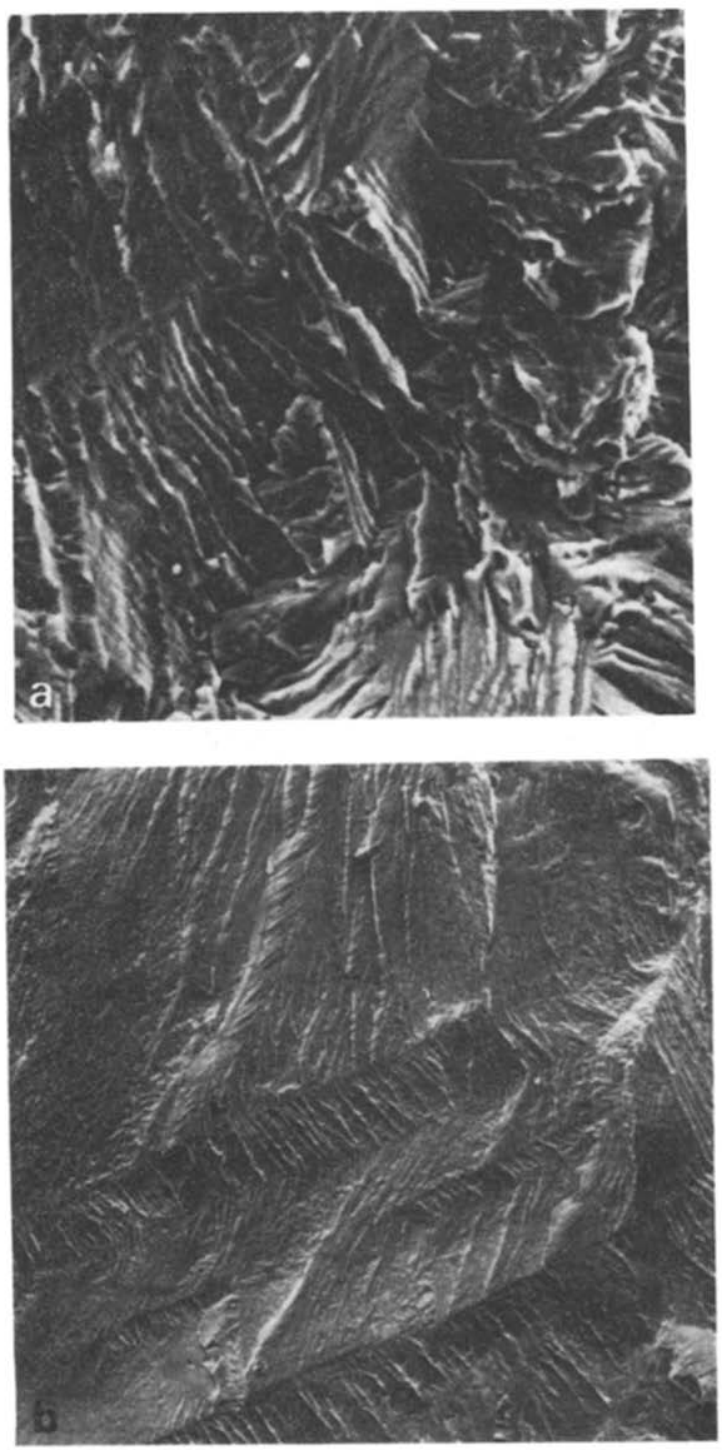

Fig. 3. Fractographic examination: (a) cyclic cleavage and plasticity tear; and (b) crack propagation through twin.

shown in $[5,7,8]$. The physical significance of the dimensionless relation $n \Delta k_{\mathrm{th}} / 2 \sigma_{\mathrm{ys}} \sqrt{d}$ is that fatigue crack starts to grow as the square root of ratio of reversed plastic zone size to grain size attains a constant value. This constant value depends on material. It is 0.6 for material tested. Mean stress ratio and crack closure effects on grain size require additional studies.

\section{Conclusions}

(1) Experimental results show that the threshold value, $\Delta k_{\mathrm{th}}$ increases linearly with the square root of the grain size and the rate is slower as grain size is increased.

(2) A dimensionless relation is suggested for collating fatigue crack starting data. The value of this relation is nearly constant for the grain sizes studied.

(3) Experimental observation shows that crack growth near threshold in Austenitic stainless steel is transgranular in form involving shear mode cyclic cleavage and plasticity tear.

\section{References}

[1] Z. Zhemin, "Continuum mechanics and fracture", $A d v$. Mech. 12(2) p. 133 (1982).

[2] Y. Nakai and T. Tanaka, "The effect of stress ratio and grain size on near threshold fatigue crack growth in lowcarbon steel", Engrg. Fracture Mech. 15(3,4), p. 291 (1981).

[3] Y. Higo, A.C. Pickard and J.F. Knott, "The effects of grain size and stacking fault energy on fatigue crack propagation threshold in Cu-Al aluminium alloys", Metal Sci. 15, p. 233 (1981).

[4] C. Robin and G. Pluvinage, "Effect of microstructure on near-threshold fatigue crack growth in 2618A aluminium alloy", Materials and Structures 3, p. 287 (1980).

[5] R. Deng, H. Li and G. Yu, "Study of plastic zone and fatigue crack propagation mechanism", Acta Metallurgica Sinica 20(2), p. A76 (1984).

[6] S. Taira, K. Tanaka and Y. Nakai, "A model of crack tip slip band blocked by grain boundary", Mech. Res. Comm. 5(6), p. 375 (1978).

[7] G.T. Hahn, R.G. Hoagland and A.R. Rosenfield, "Local yielding attending fatigue crack growth", Metall. Trans. 3(5), p. 1189 (1972).

[8] M. Clavel, D. Fournier and A. Pineau, "Plastic zone sizes in fatigued specimens of INCO 718", Metall. Trans. 6, p. 2305 (1975). 